\title{
Winding snakes in flowing nematic liquid crystals: chiral structures built from achiral building blocks
}

Qing Zhang ${ }^{\text {a }}$, Rui Zhang ${ }^{b}$, Shuang Zhouc, Irmgard Bischofberger ${ }^{\mathrm{a}}$ aMIT bHKUST cUMass Amherst

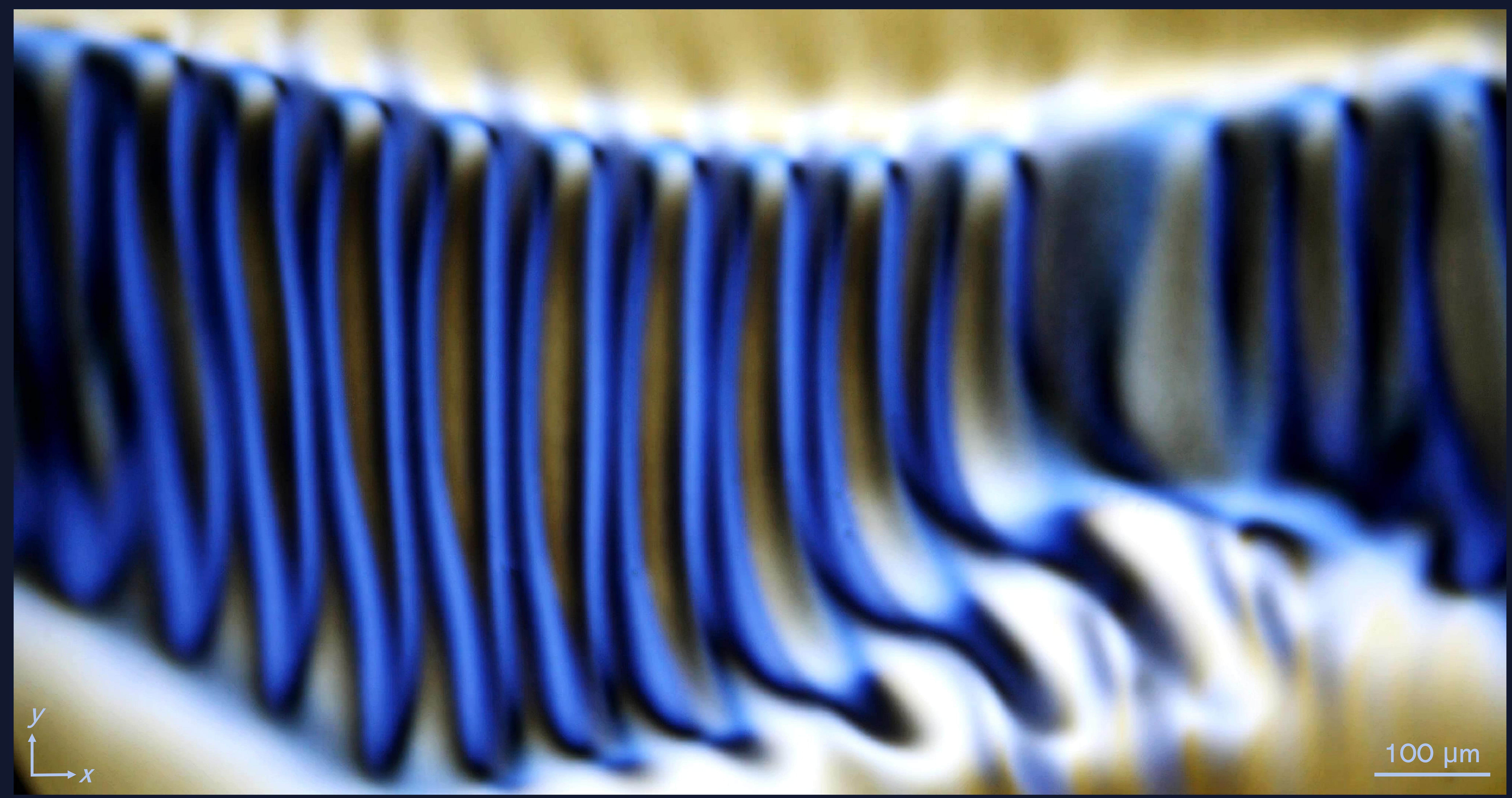

When an achiral nematic liquid crystal flows in a microfluidic channel, a snake-like structure emerges.

The structure originates from a periodic double-twist deformation of the liquid crystal; the liquid crystal exhibits twist deformations both in the flow direction $(x)$ and the gap direction ( $z$ ).

The resulting structure breaks symmetry: It is chiral structure built by achiral units; a rare phenomenon.

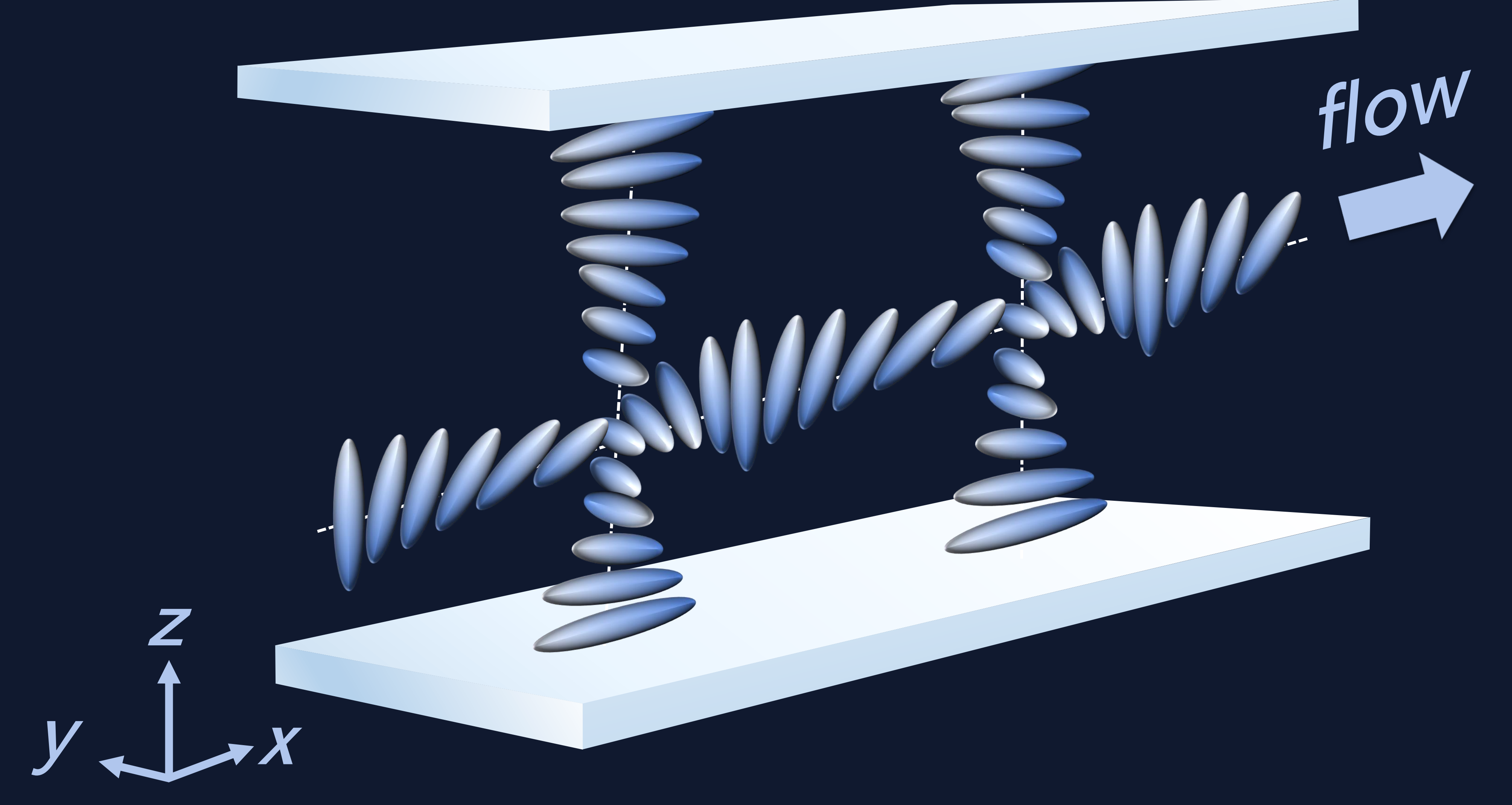

